\title{
Outage Probability Analysis of Multi-Relay Delay-Limited Hybrid-ARQ Channels (Invited Paper)
}

\author{
Behrouz Maham $^{\dagger}$, Are Hjørungnes ${ }^{\dagger}$, and Mérouane Debbah ${ }^{\ddagger}$ \\ UNIK - University Graduate Center, University of Oslo, Norway \\ ¥Alcatel-Lucent Chair on Flexible Radio, SUPÉLEC, Gif-sur-Yvette, France \\ Email: behrouz@unik.no,arehj@unik.no,merouane.debbah@supelec.fr
}

\begin{abstract}
We consider a wireless relay network with with hybrid-automatic retransmission request (HARQ) and Rayleigh fading channels. In this paper, we analyze the outage probability of the multi-relay delay-limited HARQ system with opportunistic relaying scheme in decode-and-forward mode. A simple and distributed relay selection strategy is proposed for multi-relay HARQ channels. Then, we analyze the performance of the system. We first derive the cumulative density function (CDF) and probability density function (PDF) of the selected relay channels. Then, the CDF and PDF are used to determine the outage probability in the $l$-th round of HARQ. The packet delay constraint is represented by $L$, the maximum number of HARQ rounds. Furthermore, closed-form upper-bounds on outage probability are derived, which are used to investigate the diversity order of the system. Based on the derived upper-bound expressions, it is shown that the proposed schemes achieve the full spatial diversity order of $N+1$, where $N$ is the number of potential relays. Our analytical results are confirmed by simulation results.
\end{abstract}

\section{INTRODUCTION}

Cooperation among devices has been considered to provide diversity in wireless networks where fading may significantly affect single links [1]. Initial works have emphasized on relaying, where a cooperator node amplifies (or decodes) and forwards, possibly in a quantized fashion [2], the information from the source node in order to help decoding at the destination node [3-5]. The achieved throughput can be increased with the integration of cooperation and coding, i.e., by letting the cooperator send incremental redundancy to the destination [6]. Resource allocation for space-time coded cooperative networks has been studied in [7], where an analysis of bit error rate is also derived.

A diversity effect can be introduced to a relay networks by simply allowing the nodes to maintain previously received information concerning each active message. Each time a message is retransmitted, either from a new node or from the same node, every node in the relay network will increase the amount of resolution information it has about the message. Once a node has accumulated sufficient information it will be able to decode the message and can act as a relay and forward the message (as in decode-and-forward [3], [8]). This diversity effect can be viewed as a space-time generalization of the timediversity effect of hybrid-automatic repeat request (HARQ) as

This work was supported by the Research Council of Norway through the project 176773/S10 entitled "Optimized Heterogeneous Multiuser MIMO Networks - OptiMO" and the AURORA project entitled "Communications under uncertain topologies". described in [9]. Thus, the HARQ scheme used in this paper is a practical approach to designing wireless ad hoc networks that exploit the spatial diversity, which is achievable with relaying. The retransmitted packets could originate from any node that has overheard and successfully decoded the message. Current and future wireless networks based on packet switching use HARQ protocols at the link layer. Hence, the performance of HARQ protocols in relay channels has attracted recent research interest [10-12].

In this paper, we propose an efficient HARQ multi-relay protocol which leads to full spatial diversity. We assume a multi-relay delay-limited network with the maximum number of HARQ rounds $L$, which represents the delay constraint. The closed-form expressions are derived for the outage probability, defined as the probability of packet failure after $L$ HARQ rounds, in half-duplex. Furthermore, we obtain closed-form upper-bounds for the outage probability in the high SNR scenario, from which we find the diversity order of system $N+1$, where $N$ is the number of the relays. We also propose a distributed relay selection scheme for HARQ multirelay networks by using acknowledgment (ACK) or nonacknowledgment (NACK) signals transmitted by destination.

\section{System Model AND Protocol Description}

Consider a network consisting of a source denoted $s$, one or more relays denoted $i=1,2, \ldots, N$, and one destination denoted $d$. It is assumed that each node is equipped with a single antenna. We consider symmetric channels and denote the source-to-destination, source-to- $i$ th relay, and $i$ th relayto-destination links by $f_{0}, f_{i}$, and $g_{i}$, respectively. Suppose each link has Rayleigh fading, independent of the others. Therefore, $f_{0}, f_{i}$, and $g_{i}$ are i.i.d. complex Gaussian random variables with zero-mean and variances $\sigma_{0}^{2}, \sigma_{f_{i}}^{2}$, and $\sigma_{g_{i}}^{2}$, respectively. As in [12], all links are assumed to be longterm quasi-static wherein all HARQ rounds of a single packet experience a single channel realization. Subsequent packets experience independent channel realizations. Note that such an assumption is applicable in low-mobility environments such as indoor wireless local area networks (WLANs). This clearly reveals the gains due to HARQ since temporal diversity is not present.

In this paper, we use selection relaying, a.k.a. opportunistic relaying [13], which selects the best relay among $N$ available relays. Inspired by the distributed algorithm proposed in [13], which uses request-to-send (RTS) and clear-to-send (CTS) 
signals to select the best relay, we propose the following selection procedure for HARQ systems:

- In the first step, the source node broadcast its packet toward the relays and the destination. Thus, relays can estimate their source-to-relay channels.

- Then, relays exploit the ACK or NACK signals which is transmitted by the destination to estimate their corresponding relay-to-destination channel.

- The $i$ th relay, $i=1, \ldots, N$ has a timer $T_{i}$ which its value is proportional to the inverse of $\min \left\{\left|f_{i}\right|^{2},\left|g_{i}\right|^{2}\right\}$.

- The relay with maximum amount of $\min \left\{\left|f_{i}\right|^{2},\left|g_{i}\right|^{2}\right\}$ has a smallest $T_{i}$. Whenever the first relay finished its timer, it broadcasts a flag packet toward the other relays to make them silent and announce her as the selected relay.

Let $s$ and $\hat{s}$ denote the transmitted signals from the source and the selected relay, respectively. During the first HARQ round, the relay and destination listen to the source transmit block $s$. At the end of the transmission, the destination sends both the source and relays a one-bit ACK or NACK indicating, respectively, the success or failure of the transmission. The NACK/ACK is assumed to be received error-free and with negligible delay. As long as NACK is received after each HARQ round and the maximum number of HARQ rounds is not reached, the source successively transmits subsequent HARQ blocks of the same packet. Suppose the selected relay decodes the message after HARQ round $k$, while the destination has not yet decoded the message correctly. For all HARQ rounds $l>k$, the source and the selected relay simultaneously transmit $s$ and $\hat{s}$, respectively. The Alamouti code is used to transmit the coded symbols, hence, no interference occurs due to the simultaneous transmissions of the source and relay. The effective coding rate after $l$ HARQ rounds is $R / l \mathrm{bps} / \mathrm{Hz}$, where $R$ is the spectral efficiency (in bps/Hz) of the first HARQ round. The received signal $y$ at the destination can be written as follows:

$$
y=\left\{\begin{array}{cl}
f_{0} s+g_{r} \hat{s}+n, & \text { if } l>k \\
f_{0} s+n, & \text { if } l \leq k
\end{array}\right.
$$

where the index $r$ refers to the index of the selected relay and $n$ is a complex white Gaussian noise sample with variance $N_{0}$.

\section{Outage Probability Analysis}

The recently proposed delay-limited (DL) throughput $\bar{G}_{\mathrm{DL}}$ is defined as [12]

$$
\bar{G}_{\mathrm{DL}}=\sum_{l=1}^{L} \frac{R}{l}\left[P_{\text {out }}(l-1)-P_{\text {out }}(l)\right],
$$

where $P_{\text {out }}(l)$ denotes the probability that the packet is incorrectly decoded at the destination after $l$ HARQ rounds. An advantage of definition (2), which does not resort to longterm behavior, is the ability to track slow time variations in the channels.

In [14], the request to an automatic repeat request (ARQ) is served by the relay closest to the destination, among those that have decoded the message. However, distance-dependent relay selection does not consider the fading effect of wireless networks and leads to a maximum diversity of two. Therefore, in this work, the request to an automatic repeat request (ARQ) is served by the relay with the best instantaneous channel conditions. Similar to [13], we choose the relay with the maximum of $\min \left\{\gamma_{f_{i}}, \gamma_{g_{i}}\right\}, i=1, \ldots, N$, as the best relay, where $\gamma_{f_{i}}=\left|f_{i}\right|^{2}$ and $\gamma_{g_{i}}=\left|g_{i}\right|^{2}$. We define

$$
\begin{aligned}
\gamma_{\max } & \triangleq \min \left\{\gamma_{f_{r}}, \gamma_{g_{r}}\right\} \\
& =\max \left\{\min \left\{\gamma_{f_{1}}, \gamma_{g_{1}}\right\}, \ldots, \min \left\{\gamma_{f_{N}}, \gamma_{g_{N}}\right\}\right\},
\end{aligned}
$$

where

$$
r=\arg \max _{i=1, \ldots, N}\left\{\min \left\{\gamma_{f_{i}}, \gamma_{g_{i}}\right\}\right\} .
$$

Let $\chi$ denote the earliest HARQ round after which the relay stops listening to the current message. The outage probability for the relay channel after $l$ HARQ rounds is given by [10]

$$
\begin{aligned}
P_{\text {out }}(l)= & \sum_{k=1}^{l-1} P_{\text {out }}(l \mid l>k) \operatorname{Pr}[\chi=k] \\
& +\sum_{k=l}^{L} P_{\text {out }}(l \mid l \leq k) \operatorname{Pr}[\chi=k] .
\end{aligned}
$$

To compute $\operatorname{Pr}[\chi=k]$, the mutual information between source and relay for each HARQ round is given by

$$
I_{f_{r}}=\log _{2}\left(1+\frac{P}{N_{0}} \gamma_{f_{r}}\right)
$$

where $P$ is the average transmit power from the source and $\gamma_{f_{r}}$ is an exponentially distributed random variable with mean $\sigma_{f_{r}}^{2}$. For $k=1, \ldots, l-1, \chi=k$ if the message is successfully decoded by the relay at the $k$ th HARQ round, and we have

$$
\begin{aligned}
\operatorname{Pr}[\chi=k] & =\operatorname{Pr}\left[(k-1) I_{f_{r}}<R, k I_{f_{r}}>R\right] \\
& =\operatorname{Pr}\left[(k-1) I_{f_{r}}<R\right]-\operatorname{Pr}\left[k I_{f_{r}}<R\right] \\
& =\operatorname{Pr}\left[\gamma_{f_{r}}<\mu_{k-1}\right]-\operatorname{Pr}\left[\gamma_{f_{r}}<\mu_{k}\right],
\end{aligned}
$$

where

$$
\mu_{k}=\frac{N_{0}}{P}\left(2^{R / k}-1\right) .
$$

For $k=l, \ldots, L, \chi=k$ if the relay did not decode the message successfully after $(l-1)$ HARQ rounds, and thus, we have

$$
\operatorname{Pr}[\chi=k]=\operatorname{Pr}\left[(l-1) I_{f_{r}}<R\right]=\operatorname{Pr}\left[\gamma_{f_{r}}<\mu_{l-1}\right] .
$$

From (7) and (9), $\operatorname{Pr}[\chi=k]$ can be calculated as

$$
\operatorname{Pr}[\chi=k]=\left\{\begin{array}{cl}
\operatorname{Pr}\left[\gamma_{f_{r}}<\mu_{k-1}\right]-\operatorname{Pr}\left[\gamma_{f_{r}}<\mu_{k}\right], & \text { if } k<l, \\
\operatorname{Pr}\left[\gamma_{f_{r}}<\mu_{l-1}\right], & \text { if } k \geq l .
\end{array}\right.
$$

\section{A. Approximate Outage Probability}

Since the index $r$ given in (4) is dependent on channels, $\gamma_{f_{r}}$ and $\gamma_{g_{r}}$ are not independent for $N>1$. Thus, obtaining a closed-form for PDF is not straightforward, and experimental methods can be used for finding the CDF of $\gamma_{f_{r}}$, which is required in (10). However, in the following, an approximation of the CDF of the random variable $\gamma_{f_{r}}$ is derived. 
Proposition 1: Let $\gamma_{f_{i}}$ and $\gamma_{g_{i}}, i=1, \ldots, N$, be set of independence exponential random variables with mean $\sigma_{f_{i}}^{2}=$ $\sigma_{g_{i}}^{2}=\sigma_{i}^{2}$. The cumulative density function of $\gamma_{f_{r}}$, where $r$ is defined as (4), can be approximated as

$$
\operatorname{Pr}\left\{\gamma_{f_{r}}<\gamma\right\} \approx 1-\sqrt{1-\prod_{i=1}^{N}\left(1-e^{-\frac{2 \gamma}{\sigma_{i}^{2}}}\right) .}
$$

Proof: For deriving the CDF of $\gamma_{f_{r}}$, we should first find the CDF of $\gamma_{\max }$, which can be written as

$$
\operatorname{Pr}\left\{\gamma_{\max }<\gamma\right\}=\operatorname{Pr}\left\{\gamma_{1}<\gamma, \gamma_{2}<\gamma, \ldots, \gamma_{N}<\gamma\right\}
$$

where $\gamma_{i}=\min \left\{\gamma_{f_{i}}, \gamma_{g_{i}}\right\}$ is again an exponential random variable (RV) with the parameter equal to the sum of parameters of exponential $\mathrm{RV} \gamma_{f_{i}}$ and $\gamma_{g_{i}}$, i.e., $1 / \sigma_{f_{i}}^{2}$ and $1 / \sigma_{g_{i}}^{2}$, respectively.

Thus, assuming that all channel coefficients are independent of each others, we can rewrite (12) as

$$
\operatorname{Pr}\left\{\gamma_{\max }<\gamma\right\}=\prod_{i=1}^{N}\left(1-e^{-\gamma\left(\frac{1}{\sigma_{f_{i}}^{2}}+\frac{1}{\sigma_{g_{i}}^{2}}\right)}\right) .
$$

On the other hand, we have

$$
\begin{aligned}
& \operatorname{Pr}\left\{\gamma_{\max }<\gamma\right\}=1-\operatorname{Pr}\left\{\min \left\{\gamma_{f_{r}}, \gamma_{g_{r}}\right\}>\gamma\right\} \\
& =1-\operatorname{Pr}\left\{\gamma_{f_{r}}>\gamma, \gamma_{g_{r}}>\gamma\right\} \approx 1-\operatorname{Pr}\left\{\gamma_{f_{r}}>\gamma\right\} \operatorname{Pr}\left\{\gamma_{g_{r}}>\gamma\right\},
\end{aligned}
$$

where the last equality is an approximation as if $\gamma_{f_{r}}$ and $\gamma_{g_{r}}$ are independent. For simplicity, we assume equidistance source-relay and relay-destination links, i.e., that $\sigma_{f_{i}}^{2}=\sigma_{g_{i}}^{2}=$ $\sigma_{i}^{2}$. Since we have assumed that $\gamma_{f_{i}}$ and $\gamma_{g_{i}}$ have the same statistics, using (13) and (14), we have

$$
\operatorname{Pr}\left\{\gamma_{f_{r}}<\gamma\right\}=\operatorname{Pr}\left\{\gamma_{g_{r}}<\gamma\right\} \approx 1-\sqrt{1-\prod_{i=1}^{N}\left(1-e^{-\frac{2 \gamma}{\sigma_{i}^{2}}}\right) .}
$$

In Fig. 1, we have compared the approximated PDF of $\gamma_{f_{r}}$, which is obtained by the derivation of CDF in (11), with the simulated PDF of $\gamma_{f_{r}}$. As it can be seen from Fig. 1, for the case of a single-relay network $(N=1)$, the analytical and simulated results have the same performance. This is because of the fact that the independence assumption for $\gamma_{f_{i}}$ and $\gamma_{g_{i}}$ becomes valid for $N=1$, and the approximation in (14) turns into equality. For the opportunistic relaying case, i.e., $N>1$, it can be seen that the analytical curves appropriately approximate the simulation result.

From (11), $\operatorname{Pr}[\chi=k]$ in (10) can be approximated as

$$
\begin{aligned}
\operatorname{Pr}\left[T_{r}=k\right] & \approx \sqrt{1-\prod_{i=1}^{N}\left(1-e^{-\frac{2 \mu_{k}}{\sigma_{i}^{2}}}\right)} \\
& -\sqrt{1-\prod_{i=1}^{N}\left(1-e^{-\frac{2 \mu_{k}-1}{\sigma_{i}^{2}}}\right)} \triangleq \Omega_{1}(k),
\end{aligned}
$$

for $k<l$, and

$$
\operatorname{Pr}\left[T_{r}=k\right] \approx 1-\sqrt{1-\prod_{i=1}^{N}\left(1-e^{-\frac{2 \mu_{l-1}}{\sigma_{i}^{2}}}\right)} \triangleq \Omega_{2}(l),
$$

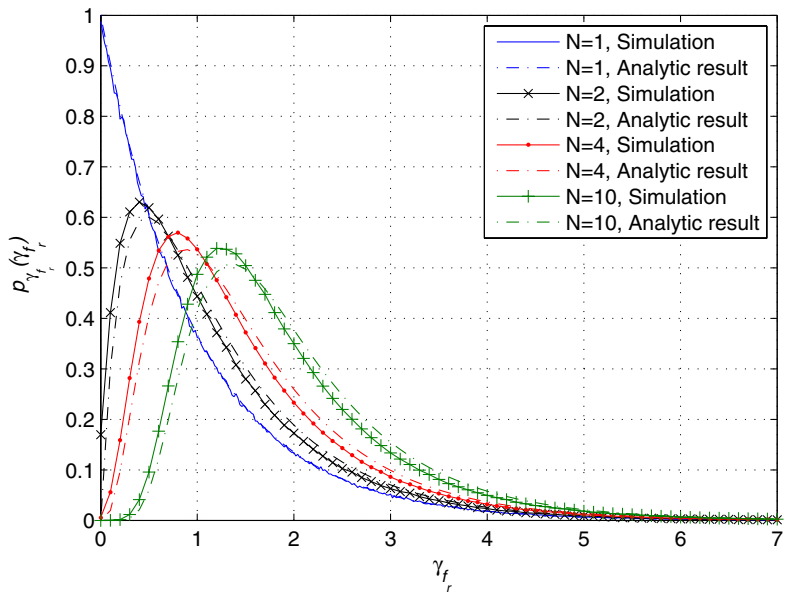

Fig. 1. Wireless multihop network under $m$-cooperation.

Next, the conditional probabilities $P_{\text {out }}(l \mid l>k)$ and $P_{\text {out }}(l \mid l \leq k)$ in (7) will be calculated.

After correct decoding of the source packet at the relay, the relay helps the source by simultaneous transmission according to the Alamouti code. Hence, assuming the relay transmits the same power $P$ as the source, the mutual information of the effective channel is given by

$$
I_{s, r, d}=\log _{2}\left(1+\frac{P}{N_{0}} \gamma_{f_{0}}+\frac{P}{N_{0}} \gamma_{g_{r}}\right) .
$$

Let $I_{\text {tot }, k, l}$ denote the total mutual information accumulated at the destination after $l$ HARQ rounds and when $\chi=k$. For $k<l$, the relay listens for $k$ HARQ rounds and transmits the message simultaneously with the source using the Alamouti code for the remaining $(l-k)$ HARQ rounds. For $k \geq l$, the relay does not help the source during the $l$ HARQ rounds. Hence,

$$
I_{\mathrm{tot}, k, l}=\left\{\begin{array}{cc}
k I_{f_{0}}+(l-k) I_{s, r, d}, & \text { if } k=1, \ldots, l-1, \\
l I_{f_{0}}, & \text { if } k=l, \ldots, L,
\end{array}\right.
$$

where $I_{f_{0}}$ is the mutual information between the source and destination at each HARQ round and can be written as $I_{f_{0}}=$ $\log _{2}\left(1+\frac{P}{N_{0}} \gamma_{f_{0}}\right)$.

Therefore, for $k \geq l$, we have

$$
P_{\text {out }}(l \mid l \leq k)=\operatorname{Pr}\left[l I_{f_{0}}<R\right]=1-\exp \left(\frac{-\mu_{l}}{\sigma_{f_{0}}^{2}}\right) .
$$

From (19), the conditional probability $P_{\text {out }}(l \mid l>k)$ can be calculated as 


$$
\begin{aligned}
& P_{\text {out }}(l \mid l>k)=\operatorname{Pr}\left[I_{\text {tot }, k, l}<R\right] \\
& =\operatorname{Pr}\left\{\log _{2}\left[\left(1+\frac{P}{N_{0}} \gamma_{f_{0}}\right)^{k}\left(1+\frac{P}{N_{0}} \gamma_{f_{0}}+\frac{P}{N_{0}} \gamma_{g_{r}}\right)^{l-k}<R\right\}\right. \\
& =\operatorname{Pr}\left\{\gamma_{g_{r}}<\frac{2^{R /(l-k)}}{\frac{P}{N_{0}}\left(1+\frac{P}{N_{0}} \gamma_{f_{0}}\right)^{k /(l-k)}}-\gamma_{f_{0}}-\frac{N_{0}}{P}\right\} \\
& =\int_{\gamma_{f_{0}}=0}^{\mu_{l}} \int_{\gamma_{g_{r}}=0}^{\beta\left(\gamma_{f_{0}}\right)} \frac{e^{-\frac{\gamma_{f_{0}}}{\sigma_{f_{0}}^{2}}}}{\sigma_{f_{0}}^{2}} p_{\gamma_{g_{r}}}\left(\gamma_{g_{r}}\right) d \gamma_{f_{0}} d \gamma_{g_{r}} \triangleq \Upsilon(l, k),
\end{aligned}
$$

where $\beta\left(\gamma_{f_{0}}\right)=\frac{2^{R /(l-k)} N_{0}}{P\left(1+\frac{P}{N_{0}} \gamma_{f_{0}}\right)^{k /(l-k)}}-\gamma_{f_{0}}-\frac{N_{0}}{P}$. Due to symmetry, the PDF of random variable $\gamma_{g_{r}}$, i.e., $p_{\gamma_{g_{r}}}(\gamma)$, is same as the PDF of random variable $\gamma_{g_{r}}$, with perhaps different mean. Thus, the PDF of $\gamma_{g_{r}}$ can be found by the derivation of $\operatorname{Pr}\left\{\gamma_{g_{r}}<\gamma\right\}$ in (15) as

$$
p_{\gamma_{g_{r}}}(\gamma) \approx \frac{1}{\sqrt{1-\prod_{i=1}^{N}\left(1-e^{-\frac{2 \gamma}{\sigma_{i}^{2}}}\right)}} \sum_{i=1}^{N} \frac{e^{-\frac{2 \gamma}{\sigma_{i}^{2}}}}{\sigma_{i}^{2}} \prod_{\substack{j=1 \\ j \neq i}}^{N}\left(1-e^{-\frac{2 \gamma}{\sigma_{j}^{2}}}\right) .
$$

By substituting $p_{\gamma_{g_{r}}}(\gamma)$ from (22) into (21), $P_{\text {out }}(l \mid l>k)$ is obtained. Therefore, using (10), (20), and (21), the outage probability in the $l$ th stage of HARQ process can be achieved as

$$
P_{\text {out }}(l) \approx \sum_{k=1}^{l-1} \Omega_{1}(k) \Upsilon(l, k)+\sum_{k=l}^{L} \Omega_{2}(l)\left(1-e^{\frac{-\mu_{l}}{\sigma_{f}^{l}}}\right) .
$$

\section{B. Upper-Bound on Outage Probability}

For calculating the minimum diversity gain of HARQ wireless relay networks when selection strategy in (4) is used, it is enough to derive an upper-bound on the outage probability $P_{\text {out }}(l)$.

The random variable $\gamma_{f_{r}}$, which is corresponding the source-relay channel of the selected relay, can be bounded as

$$
\gamma_{\max } \leq \gamma_{f_{r}} \leq \gamma_{\max }^{s},
$$

where $\gamma_{\max }$ is given in (3) and $\gamma_{\max }^{s}$ is defined as

$$
\gamma_{\max }^{s}=\max _{i=1, \ldots, N}\left\{\gamma_{f_{i}}\right\} \text {. }
$$

The CDF of $\gamma_{\max }^{s}$ can be written as

$$
\begin{aligned}
\operatorname{Pr}\left\{\gamma_{\max }^{s}<\gamma\right\} & =\operatorname{Pr}\left\{\gamma_{f_{1}}<\gamma, \gamma_{f_{2}}<\gamma, \ldots, \gamma_{f_{N}}<\gamma\right\} \\
& =\prod_{i=1}^{N}\left(1-e^{-\frac{\gamma}{\sigma_{f_{i}}^{2}}}\right) .
\end{aligned}
$$

Thus, it is easy to show that the $\mathrm{CDF}$ of $\gamma_{\max }^{s}$ can be written as

$$
\operatorname{Pr}\left\{\gamma_{\text {max }}^{s}<\gamma\right\} \leq \operatorname{Pr}\left\{\gamma_{f_{r}}<\gamma\right\} \leq \operatorname{Pr}\left\{\gamma_{\max }<\gamma\right\} .
$$

Therefore, an upper-bound on $\operatorname{Pr}[\chi=k]$ will be obtained as follows: Combining (10) and (27), we have

$$
\operatorname{Pr}[\chi=k] \leq \operatorname{Pr}\left[\gamma_{\max }<\mu_{k-1}\right]-\operatorname{Pr}\left[\gamma_{\max }^{s}<\mu_{k}\right],
$$

for $k<l$. From (13), (26), and (28), $\operatorname{Pr}[\chi=k]$ for $k<l$ can be calculated as

$$
\begin{aligned}
\operatorname{Pr}[\chi=k] & \leq \prod_{i=1}^{N}\left(1-e^{-\mu_{k-1}\left(\frac{1}{\sigma_{f_{i}}^{2}}+\frac{1}{\sigma_{g_{i}}^{2}}\right)}\right)-\prod_{i=1}^{N}\left(1-e^{-\frac{\mu_{k}}{\sigma_{f_{i}}^{2}}}\right) \\
& \triangleq \Lambda_{1}(k) .
\end{aligned}
$$

For $k \geq l$, by combining (10), (13), and (27), we have

$$
\begin{aligned}
& \operatorname{Pr}[\chi=k] \leq \operatorname{Pr}\left[\gamma_{\max }<\mu_{l-1}\right] \\
& =\prod_{i=1}^{N}\left(1-e^{-\mu_{l-1}\left(\frac{1}{\sigma_{f_{i}}^{2}}+\frac{1}{\sigma_{g_{i}}^{2}}\right)}\right) \triangleq \Lambda_{2}(l) .
\end{aligned}
$$

Next, $P_{\text {out }}(l \mid l>k)$ in (21) can be upper-bounded as

$$
P_{\text {out }}(l \mid l>k)
$$

$$
\begin{aligned}
& \leq \operatorname{Pr}\left\{\gamma_{\max }<\frac{2^{R /(l-k)}}{\frac{P}{N_{0}}\left(1+\frac{P}{N_{0}} \gamma_{f_{0}}\right)^{k /(l-k)}}-\gamma_{f_{0}}-\frac{N_{0}}{P}\right\} \\
& =\int_{\gamma_{f_{0}=0}}^{\mu_{l}} \int_{\gamma_{\max }=0}^{\beta\left(\gamma_{f_{0}}\right)} \frac{e^{-\frac{\gamma_{f_{0}}}{\sigma_{f_{0}}^{2}}}}{\sigma_{f_{0}}^{2}} p_{\gamma_{\max }}\left(\gamma_{\max }\right) d \gamma_{f_{0}} d \gamma_{\max } .
\end{aligned}
$$

The PDF of random variable $\gamma_{\max }$, i.e., $p_{\gamma_{\max }}(\gamma)$ can be found by the derivative of $\operatorname{Pr}\left\{\gamma_{\max }<\gamma\right\}$ in (13). Thus, we have

$$
\begin{aligned}
p_{\gamma_{\max }}(\gamma) & =\sum_{i=1}^{N}\left(\frac{1}{\sigma_{f_{i}}^{2}}+\frac{1}{\sigma_{g_{i}}^{2}}\right) e^{-\gamma\left(\frac{1}{\sigma_{f_{i}}^{2}}+\frac{1}{\sigma_{g_{i}}^{2}}\right)} \\
& \times \prod_{\substack{j=1 \\
j \neq i}}^{N}\left(1-e^{-\gamma\left(\frac{1}{\sigma_{f_{j}}^{2}}+\frac{1}{\sigma_{g_{j}}^{2}}\right)}\right) .
\end{aligned}
$$

Therefore, by substituting $\operatorname{Pr}[\chi=k]$ from (29) and (30), and $P_{\text {out }}(l \mid l \leq k)$ and $P_{\text {out }}(l \mid l>k)$ from (20) and (31), respectively, in (5), an upper-bound on outage probability the $l$ th stage of HARQ process, i.e., $P_{\text {out }}(l)$ can be achieved.

A tractable definition of the diversity gain is [15, Eq. (1.19)]

$$
G_{d}=-\lim _{\rho \rightarrow \infty} \frac{\log \left(P_{\text {out }}\right)}{\log (\rho)},
$$

where $\rho=\frac{P}{N_{0}}$. Thus, in the following, we investigate the asymptotic behavior and diversity order of $P_{\text {out }}(l)$ in (5).

From (32), an upper-bound for $p_{\gamma_{\max }}(\gamma)$ can be found as

$$
p_{\gamma_{\max }}(\gamma) \leq N \gamma^{N-1} \prod_{i=1}^{N}\left(\frac{1}{\sigma_{f_{i}}^{2}}+\frac{1}{\sigma_{g_{i}}^{2}}\right),
$$

which is a tight bound when $\gamma \stackrel{i=1}{\rightarrow} 0$. Note that in high SNR scenario, the the behavior of the fading distribution around zero is important (see, e.g., [16]).

Using (34) and the fact that the exponential distribution is a decreasing function of $\gamma_{f_{0}}, P_{\text {out }}(l \mid l>k)$ in (31) can be further upper-bounded as

$P_{\text {out }}(l \mid l>k)$

$$
\begin{aligned}
& \leq \int_{\gamma_{f_{0}}=0}^{\mu_{l}} \int_{\gamma_{\max }=0}^{\beta\left(\gamma_{f_{0}}\right)} \frac{1}{\sigma_{f_{0}}^{2}} \gamma_{\max }^{N-1} \prod_{i=1}^{N}\left(\frac{1}{\sigma_{f_{i}}^{2}}+\frac{1}{\sigma_{g_{i}}^{2}}\right) d \gamma_{f_{0}} d \gamma_{\max } \\
& \leq \frac{\mu_{l}}{\sigma_{f_{0}}^{2}} \mu_{l-k}^{N} \prod_{i=1}^{N}\left(\frac{1}{\sigma_{f_{i}}^{2}}+\frac{1}{\sigma_{g_{i}}^{2}}\right) \triangleq \Psi(l, k)
\end{aligned}
$$


Combining (5), (20), (29), (30), and (36), a closed-form upperbound for the outage probability after $l$ HARQ round can be obtained as

$$
P_{\text {out }}(l) \leq \sum_{k=1}^{l-1} \Lambda_{1}(k) \Psi(l, k)+\sum_{k=l}^{L} \Lambda_{2}(l)\left(1-e^{\frac{-\mu_{l}}{\sigma_{f_{0}}^{2}}}\right) .
$$

Furthermore, using (16), and (17), another closed-form approximation for $P_{\text {out }}(l)$ can be obtained as

$$
P_{\text {out }}(l) \approx \sum_{k=1}^{l-1} \Omega_{1}(k) \Psi(l, k)+\sum_{k=l}^{L} \Omega_{2}(l)\left(1-e^{\frac{-\mu_{l}}{\sigma_{f_{0}}^{2}}}\right) .
$$

Proposition 2: Assuming a HARQ system with $N$ potential relays nodes, the relay selection strategy based on (4) can achieve the full diversity order of $N+1$.

Proof: From a Taylor series expansion, it can be shown that the first term in (36) is $O\left(1 / \rho^{2 N+1}\right)$. From (36), and by representing the factor $\mu_{k}$ in terms of the SNR ratio $\rho$, the outage probability in high SNR can be written as

$$
P_{\text {out }}(l) \leq \frac{\Delta(l)}{\rho^{N+1}},
$$

where

$\Delta(l)=\left(2^{\frac{R}{l}}-1\right)\left(2^{\frac{R}{l-1}}-1\right)^{N} \frac{L-l+1}{\sigma_{f_{0}}^{2}} \prod_{i=1}^{N}\left(\frac{1}{\sigma_{f_{i}}^{2}}+\frac{1}{\sigma_{g_{i}}^{2}}\right)$.

Hence, observing (38), the diversity order defined in (33) is equal to $N+1$, which is the full spatial diversity for $N+1$ transmitting nodes.

\section{NUMERICAL ANALYSIS}

In this section, the performance of the proposed relayselection HARQ system is studied through numerical results. We used the equal power allocation among the source and the selected relay. Assume the relays and the destination have the same value of noise power, and all the links have unitvariance Rayleigh flat fading, i.e., $\sigma_{f_{i}}^{2}=\sigma_{g_{i}}^{2}=\sigma_{f_{0}}^{2}=1$. It is also assumed that rate $R$ is normalized to 1 . We compare the transmit SNR $\frac{P}{N_{0}}$ versus outage probability performance. The block fading model is used, in which channel coefficients changed randomly in time to isolate the benefits of spatial diversity. The simulation result is averaged over 3'000'000 transmitted symbols (channel realization trials).

Fig. 2 confirms that the analytical results attained in Section III for the outage probability have an accurate performance as the simulation results. We consider the maximum number of HARQ rounds to be $L=5$. The outage probability at the 2nd HARQ round, i.e., $P_{\text {out }}(l=2)$, is compared for two different number of relays $N=2,4$. One can see the approximate outage probability derived in (23) has the similar performance as the simulated curved for all values of SNR. In addition, the closed-form outage probability expression in (37) well approximates the simulated results, especially in medium and high SNR conditions. Furthermore, Fig. 2 shows that the upper-bound expression in (36) is a tight upper-bound.

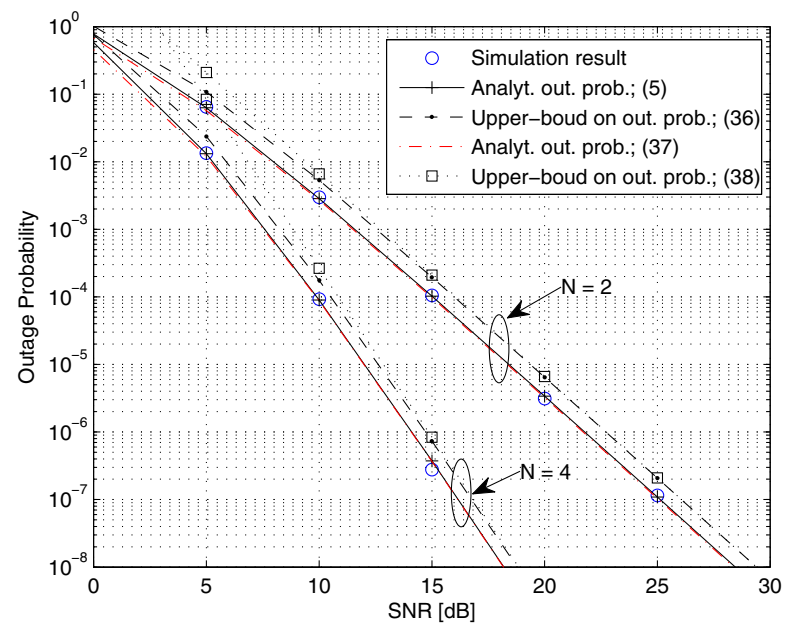

Fig. 2. The outage probability curves of delay-limited HARQ networks employing opportunistic relaying with 2 and 4 relays, when $R=1, l=2$, and $L=5$.

\section{REFERENCES}

[1] A. Nosratinia, T. Hunter, and A. Hedayat, "Cooperative communication in wireless networks," IEEE Commun. Mag., vol. 42, no. 10, pp. 74-80, Oct. 2004.

[2] T. Cover and A. Gamal, "Capacity theorems for the relay channel," IEEE Trans. Info. Theory, vol. 25, pp. 572-584, Sep. 1979.

[3] J. N. Laneman, D. Tse, and G. Wornell, "Cooperative diversity in wireless networks: Efficient protocols and outage behavior," IEEE Trans. Inform. Theory, vol. 50, no. 12, pp. 3062-3080, Dec. 2004.

[4] B. Maham, A. Hjørungnes, and G. Abreu, "Distributed GABBA spacetime codes in amplify-and-forward relay networks," IEEE Trans. Wireless Commun., vol. 8, no. 4, pp. 2036-2045, Apr. 2009.

[5] B. Maham and A. Hjørungnes, "Performance analysis of repetition-based cooperative networks with partial statistical CSI at relays," IEEE Comm. Letters, vol. 12, no. 11, pp. 828-830, Nov. 2008.

[6] T. E. Hunter, S. Sanayei, and A. Nosratinia, "Outage analysis of coded cooperation," IEEE Trans. Inform. Theory, vol. 52, no. 2, pp. 375-391, Feb. 2006.

[7] B. Maham and A. Hjørungnes, "Power allocation strategies for distributed space-time codes in amplify-and-forward mode," EURASIP Journal on Advances in Signal Processing, vol. 2009, Article ID 310247, 13 pages, 2009.

[8] B. Maham and A. Hjørungnes, "Differential space-time coded cooperation for decode-and-forward based wireless relay networks," IET Communications (former IEE Proc. COM). accepted Nov. 2009. Available online http://persons.unik.no/behrouz/documents/IET.pdf.

[9] G. Caire and D. Tuninetti, "The throughput of hybrid-ARQ protocols for the Gaussian collision channel," IEEE Trans. Inf. Theory, vol. 47, pp. 1971-1988, Jul. 2001.

[10] T. Tabet, S. Dusad, and R. Knopp, "Achievable diversity-multiplexingdelay tradeoff in half-duplex arq relay channels," in Proc. IEEE ISIT, (Adelaide, Australia), Sep. 2005.

[11] B. Zhao and M. C. Valenti, "Practical relay networks: a generalization of hybrid-ARQ," IEEE J. Select. Areas Commun., vol. 23, no. 1, pp. 7-18, Jan. 2005.

[12] R. Narasimhan, "Throughput-delay performance of half-duplex hybridARQ relay channels," in Proc. IEEE Int. Conf. Commun. (ICC), (Beijing, China), May 2008.

[13] A. Bletsas, A. Khisti, D. P. Reed, and A. Lippman, "A simple cooperative method based on network path selection," IEEE Journal on Selected Areas in Communications, vol. 24, no. 3, pp. 659-672, Mar. 2006.

[14] M. Zorzi and R. R. Rao, "Geographic random forwarding (GeRaF) for ad hoc and sensor networks: Multihop performance," IEEE Trans. Mobile Comput., vol. 2, no. 4, pp. 337-348, Oct.-Dec. 2003.

[15] H. Jafarkhani, Space-Time Coding Theory and Practice. Cambridge, UK: Cambridge Academic Press, 2005.

[16] A. Ribeiro, A. Cai, and G. B. Giannakis, "Symbol error probablity for general cooperative links," IEEE Trans. Wireless Commun., vol. 4 pp. 1264-1273, May 2005. 